\title{
Geographical distribution of Amblyomma cajennense (sensu lato) ticks (Parasitiformes: Ixodidae) in Brazil, with description of the nymph of $A$. cajennense (sensu stricto)
}

Thiago F. Martins ${ }^{1}$, Amália R. M. Barbieri ${ }^{1}$, Francisco B. Costa ${ }^{1}$, Flávio A. Terassini ${ }^{2}$, Luís M. A. Camargo ${ }^{2,3}$, Cássio R. L. Peterka ${ }^{4}$, Richard de C. Pacheco ${ }^{5}$, Ricardo A. Dias ${ }^{1}$, Pablo H. Nunes ${ }^{6}$, Arlei Marcili ${ }^{1,7}$, Alessandra Scofield ${ }^{8}$, Artur K. Campos ${ }^{9}$, Mauricio C. Horta ${ }^{10}$, Aline G. A. Guilloux ${ }^{1}$, Hector R. Benatti ${ }^{1}$, Diego G. Ramirez ${ }^{11}$, Darci M. Barros-Battesti ${ }^{11}$ and Marcelo B. Labruna ${ }^{1 *}$

\begin{abstract}
Background: Until recently, Amblyomma cajennense (Fabricius, 1787) was considered to represent a single tick species in the New World. Recent studies have split this taxon into six species. While the A. cajennense species complex or A. cajennense (sensu lato) (s.l.) is currently represented by two species in Brazil, A. cajennense (sensu stricto) (s.s.) and Amblyomma sculptum Berlese, 1888, their geographical distribution is poorly known.
\end{abstract}

Methods: The distribution of the A. cajennense (s.l.) in Brazil was determined by morphological examination of all lots of A. cajennense (s.l.) in two large tick collections of Brazil, and by collecting new material during three field expeditions in the possible transition areas between the distribution ranges of A. cajennense (s.s.) and A. sculptum. Phylogenetic analysis inferred from the ITS2 rRNA gene was used to validate morphological results. Morphological description of the nymphal stage of A. cajennense (s.s.) is provided based on laboratory-reared specimens.

Results: From the tick collections, a total 12,512 adult ticks were examined and identified as 312 A. cajennense (s.s.), 6,252 A. sculptum and 5,948 A. cajennense (s.l.). A total of 1,746 ticks from 77 localities were collected during field expeditions, and were identified as 249 A. cajennense (s.s.), 443 A. sculptum, and 1,054 A. cajennense (s.l.) [these A. cajennense (s.l.) ticks were considered to be males of either A. cajennense (s.s.) or A. sculptum]. At least 23 localities contained the presence of both A. cajennense (s.s.) and A. sculptum in sympatry. DNA sequences of the ITS2 gene of 50 ticks from 30 localities confirmed the results of the morphological analyses. The nymph of A. cajennense (s.s.) is morphologically very similar to A. sculptum.

Conclusion: Our results confirmed that A. cajennense (s.l.) is currently represented in Brazil by only two species, $A$. cajennense (s.s.) and A. sculptum. While these species have distinct distribution areas in the country, they are found in sympatry in some transition areas. The current distribution of A. cajennense (s.l.) has important implications to public health, since in Brazil A. sculptum is the most important vector of the bacterium Rickettsia rickettsii, the etiological agent of Brazilian spotted fever.

Keywords: Amblyomma cajennense, Amblyomma sculptum, Distribution, ITS2, Nymph

\footnotetext{
* Correspondence: labruna@usp.br

1Departamento de Medicina Veterinária Preventiva e Saúde Animal,

Faculdade de Medicina Veterinária e Zootecnia, Universidade de São Paulo,

Av. Prof. Orlando Marques de Paiva 87, São Paulo 05508-270, Brazil

Full list of author information is available at the end of the article
} 


\section{Background}

Until recently, the taxon Amblyomma cajennense (Fabricius, 1787) was considered to represent a single tick species occurring in southern United States, Mexico, Central America, Caribbean and all countries of South America with the exception of Chile and Uruguay [1]. A recent morphological study [2], supported by biological [3, 4] and molecular analyses [5], split this taxon into six valid species, namely A. cajennense sensu stricto (s.s.) (restricted to the Amazonian region), Amblyomma mixtum Koch, 1844 (from Texas to western Ecuador), Amblyomma sculptum Berlese, 1888 (northern Argentina, Bolivia, Paraguay, Brazil), Amblyomma interandinum Beati, Nava \& Cáceres, 2014 (inter-Andean valley of Peru), Amblyomma tonelliae Nava, Beati \& Labruna, 2014 (dry areas of northern Argentina, Bolivia and Paraguay), and Amblyomma patinoi Labruna, Nava \& Beati, 2014 (Eastern Andes of Colombia) $[2,5]$. With this new classification, the A. cajennense species complex or A. cajennense (sensu lato) (s.l.) is currently represented by two species in Brazil, A. cajennense (sensu stricto) and A. sculptum, which can be morphologically separated only by examination of the genital aperture of females [2]. However, as stated by Nava et al. [2], the known geographical distribution of these species is still incomplete. In addition, it is not known if there is any geographical overlap between $A$. cajennense (s.s.) and $A$. sculptum.

There have been four previous descriptions of nymphs of $A$. cajennense (s.l.): two of $A$. cajennense (s.l.) [6, 7], one of A. sculptum [8], and one of A. tonelliae [8]. Indeed, the description of the nymph of $A$. cajennense by Martins et al. [7] refers to A. sculptum, since the described ticks were from the state of São Paulo, southeastern Brazil, a typical area for the occurrence of A. sculptum [2]. While Cooley \& Kohls [6] did not mention the specific origin of the described nymphs, it is possible that their nymphs were $A$. mixtum, the only species of $A$. cajennense (s.l.) known to occur in North America [2].
The present study aimed to determine the geographical distribution of $A$. cajennense (s.l.) in Brazil. For this purpose, all lots of $A$. cajennense (s.l.) in two large tick collections of Brazil were revised. New material was acquired during three field expeditions in the possible transition areas between the distribution ranges of $A$. cajennense (s.s.) and A. sculptum, where these two species might overlap. In addition, molecular analyses were performed on representative tick specimens from different regions, in order to confirm their taxonomic identification. Finally, a morphological description of the nymphal stage of $A$. cajennense (s.s.) is provided for the first time.

\section{Methods}

\section{Examination of ticks deposited at tick collections}

The following tick collections were assessed: the "Acari Colletion of the Instituto Butantan" (IBSP), São Paulo, Brazil; and the "Coleção Nacional de Carrapatos" (CNC) of the University of São Paulo, São Paulo, Brazil. In each tick collection, all lots containing adults of $A$. cajennense (s.l.) were examined morphologically under a stereomicroscope. Females were identified as $A$. cajennense (s.s.) or A. sculptum, based on the genital aperture morphology (Fig. 1), i.e. V-shaped in the former and Ushaped in the latter [2]. Due to the lack of discriminating characters to separate males of A. cajennense (s.s.) and A. sculptum (see Nava et al. [2]), males were morphologically identified as A. cajennense (s.l.) From each lot, the geographical coordinates of the municipality was retrieved from the "Instituto Brasileiro de Geografia e Estatística” (IBGE) website (www.ibge.gov.br).

\section{Field expeditions for collection of ticks}

Three field expeditions were performed in the possible transition areas of the distribution ranges of $A$. cajennense (s.s.) and A. sculptum in Brazil. One expedition, during January 2012, included areas of the states of Maranhão, Pará, Tocantins, and Goiás. These states were considered to represent the eastern transition area of the

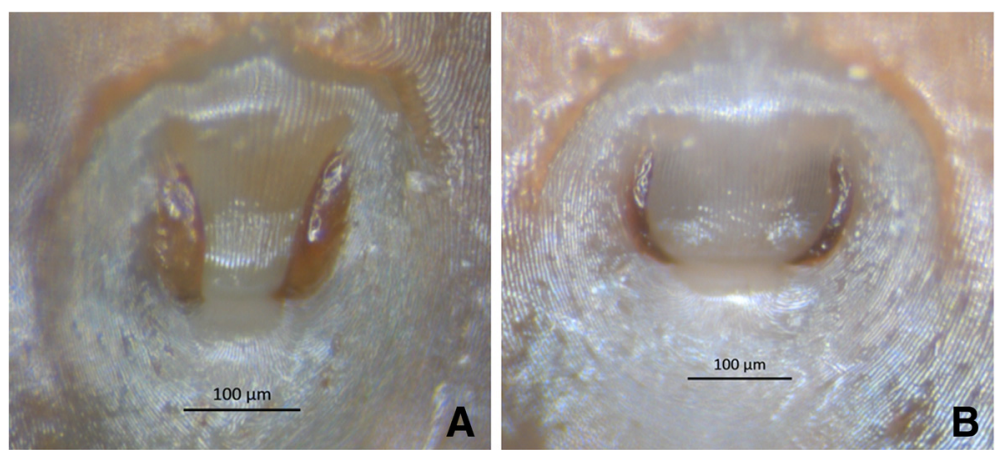

Fig. 1 Genital aperture of adult females. a "V" shaped in Amblyomma cajennense (sensu stricto), a specimen from Governador Jorge Teixeira, Rondônia, Brazil; b "U" shaped in Amblyomma sculptum, a specimen from Pirassununga, São Paulo, Brazil 
two species. A second expedition, also during January 2012, included areas of the states of Rondônia and Mato Grosso, which were considered to represent the western transition area of two species in Brazil. Finally, a third expedition, during February 2013, included the state of Mato Grosso, in areas that could represent the central transition area of the two species in Brazil. The three expeditions were carried out during January or February because previous studies indicated that these months correspond in Brazil to the highest abundance of the adult stage of A. cajennense (s.s.) [9] or A. sculptum [10]. Each expedition consisted of driving a car through regular roads and stopping at regular distances (usually every $\approx 50 \mathrm{Km}$ ) for examination of horses and pigs (main domestic hosts for A. cajennense (s.l.) in Brazil [11, 12]) for tick infestations, and/or for collecting questing ticks on the vegetation by dragging and visual search methods [13]. In addition, road-killed tapirs (Tapirus terrestris Linnaeus, 1758) and anteaters (Myrmecophaga tridactyla Linnaeus, 1758), two main hosts for A. cajennense (s.l.) $[11,12]$ were occasionally examined for tick infestations. Every time A. cajennense (s.l.) ticks were found, they were collected in plastic vials containing absolute ethanol, and the geographic coordinates were recorded with the use of a Global Position System apparatus (GPS Garmin, model GPS V, Olathe, KA, USA). All ticks were taken to the laboratory for taxonomic identification as stated above.

\section{Molecular analyses}

For molecular analyses, we used one to three specimens from each given location. From the material collected in the field expeditions, we prioritised male specimens from locations where no female specimen was available, since species identification through morphological examination can be achieved only for females. In addition, we processed male and female specimens taken from the CNC tick collection, from different locations of Brazil, in order to have representative specimens from nearly all Brazilian states where A. cajennense (s.l.) has been reported.

Each tick specimen was individually submitted to DNA extraction by the guanidine thiocyanate method [14], and tested by a PCR assay targeting the entire tick ITS2 rRNA gene, with primers corresponding to the flanking regions (5.8S rRNA 3 '-end, and $28 \mathrm{~S}$ rRNA $5^{\prime}$-end), as previously described [15]. All PCR products of the expected size $(\approx 1,100 \mathrm{bp})$ were purified with ExoSap (USB, Cleveland, OH, USA) and sequenced in an ABI automated sequencer (Applied Biosystems/Thermo Fisher Scientific, model ABI 3500 Genetic Analyser, Foster City, CA, USA) with the same primers used for PCR.

\section{Phylogenetic analysis}

The ITS2 sequences generated in the present study, excluding the rRNA $5.8 \mathrm{~S}$ and $28 \mathrm{~S}$ flanking regions, were aligned with GenBank sequences for A. sculptum from Minas Gerais, Brazil (JN866842, JN866843), São Paulo, Brazil (JN866846), and Argentina (JN866835); A. mixtum from Costa Rica (JN866853) and Mexico (JN866886); $A$. patinoi from Colombia (JN866881, JN866882); A. tonelliae from Argentina (JN866895, JN866896, JN866897); A. interandinum from Peru (JN866900, JN866902, JN866905); and A. cajennense (s.s.) from French Guiana (JN866864) $[2,5]$ using Clustal X [16], and were manually adjusted using GeneDoc, version 2.6.01 [17]. Thereafter, the ITS2 sequences of Amblyomma aureolatum (Pallas, 1772) (AF469611) and Amblyomma americanum (Linnaeus, 1758) (AF291874) were added as outgroup.

The ITS2 alignment was used to construct a phylogenetic tree using maximum parsimony, as implemented in PAUP version 4.0b10 [18] with 500 bootstrap replicates, random stepwise addition starting trees (with random addition sequences) and TBR branch swapping. Bayesian analysis was performed using MrBayes v3.1.2 [19] with four independent Markov chain runs for 5,000,000 metropolis-coupled MCMC generations, sampling a tree every $100^{\text {th }}$ generation. The first $25 \%$ of the trees represented "burn-in", and the remaining trees were used to calculate Bayesian posterior probabilities, which are presented in the consensus tree.

\section{Distribution maps}

For construction of distribution maps of A. cajennense (s.s.) and A. sculptum in Brazil, we used the geographical coordinates of all lots of these two species in the IBSP and $\mathrm{CNC}$ tick collections, plus the coordinates of the ticks collected during the three field expeditions of the present study. Tick locations were plotted on four different maps: (i) geopolitical, (ii) biomes, (iii) climate, and (iv) remaining natural vegetation cover (forest remains). Map sources used in the present study were obtained from the "Instituto Brasileiro de Geografia e Estatística" (IBGE) website (www.ibge.gov.br) and the "Ministério do Meio Ambiente"(MMA) website (www.mma.gov.br). The distribution maps were constructed with the use of ArcGIS version 10.0 (ESRI).

\section{Description of the nymph of $A$. cajennense (s.s.)}

In July 2014, a tick colony of $A$. cajennense (s.s.) was established in the laboratory, started with adult ticks collected on the vegetation at Governador Jorge Teixeira municipality $\left(10^{\circ} 31^{\prime} \mathrm{S}, 62^{\circ} 38^{\prime} \mathrm{W}\right)$, state of Rondônia, Brazil. Unfed $F_{1}$ nymphs, 15-30 day-old, were killed in hot water $\left(70-80{ }^{\circ} \mathrm{C}\right)$ and immediately preserved in $70 \%$ alcohol until further processing for description. Nymphal description, based on optical microscopy, followed Martins et al. 
[7]. Measurements were taken from 10 specimens using the programme Image-Pro Plus 5.1 for analysis of images and morphometry, fitted to an Olympus SZX stereoscope microscope. All measurements are in micrometres and are given as the range followed by the mean in parentheses. These nymphs were deposited in the CNC (accession number CNC-2913). Nymphs were prepared for scanning electron microscopy (SEM) following previously described techniques [20] and SEM micrographs were taken using a HITACHI TM3000 Scanning Electron Microscope.

\section{Ethical approval}

This work has been approved by the Ethic Committee in the Use of Animals of the Faculty of Veterinary Medicine of the University of Sao Paulo (project number 2660/2012).

\section{Results}

\section{Examination of ticks deposited at tick collections}

A total of 335 lots of adult ticks A. cajennense (s.l.) were examined from the IBSP tick collection. These were identified as 67 females of A. cajennense (s.s.), 648 females of $A$. sculptum, and 978 males of A. cajennense (s.l.) Similarly, a total of 545 lots of adult ticks A. cajennense (s.l.) were examined at the $\mathrm{CNC}$ tick collection. These were identified as 245 females of $A$. cajennense (s.s.), 5,604 females of $A$. sculptum and 4,970 males of $A$. cajennense (s.l.) For each lot examined, host, date, and locality data (including geographical coordinates) are listed in Additional file 1: Table S1).

From a total of 55 lots of $A$. cajennense (s.s.) with host data (total: at least 16 different host species), the most common hosts were Equus caballus Linnaeus, 1758 (12 lots, $21.8 \%$ ) and T. terrestris (10 lots, $18.2 \%$ ), followed by Canis lupus familiaris Linnaeus, 1758 (6 lots, 10.9 \%), Bos taurus Linnaeus, 1758 (5 lots, $9.1 \%$ ), M. tridactyla (4 lots, $7.3 \%$ ), and Homo sapiens Linnaeus, 1758 (3 lots, $5.5 \%$ ). From a total of 438 lots of A. sculptum with host data (total: at least 48 different host species), the most common hosts were $H$. sapiens (55 lots, $12.6 \%$ ) and E. caballus (54 lots, $12.3 \%$ ), followed by Hydrochoerus hydrochaeris (Linnaeus, 1766) (47 lots, $10.7 \%$ ), T. terrestris (46 lots, $10.5 \%$ ), $M$. tridactyla (26 lots, $5.9 \%$ ), Panthera onca (Linnaeus, 1758) (23 lots, $5.3 \%$ ), and C. lupus familiaris (21 lots, $4.8 \%$ ) (Additional file 1: Table S1).

\section{Ticks collected during field expeditions}

A total of 1,746 ticks from 77 localities were collected during the three field expeditions. These were morphologically identified as 249 females of $A$. cajennense (s.s.), 443 females of $A$. sculptum and 1,054 males of $A$. cajennense (s.l.) Detailed information on the ticks collected at each locality are listed in Table 1 . Some of the above male specimens were identified to A. cajennense (s.s.) or A. sculptum by molecular methods, as stated in Table 2 . At least 23 localities contained the presence of both $A$. cajennense (s.s.) and A. sculptum in sympatry, inferred by the presence of females of both species co-infesting the same individual hosts.

\section{Molecular and phylogenetic analyses}

DNA sequences of the ITS2 gene were generated for 24 A. cajennense (s.s.) from 14 different localities, and 26 A. sculptum from 16 localities (Table 2). Phylogenetic analyses of these ITS2 sequences with corresponding sequences from GenBank formed six main clades, each corresponding to a species of $A$. cajennense (s.l.): $A$. cajennense (s.s.), A. sculptum, A. mixtum, A. tonelliae, A. internadinum and $A$. patinoi (Fig. 2). There was no polymorphism among the A. cajennense (s.s.) sequences, since all 24 tick specimens shared the same haplotype, which was $100 \%$ identical to the GenBank ITS2 sequence of $A$. cajennense (s.s.) from French Guyana (JN866864). Conversely, the 26 A. sculptum presented polymorphism, as they yielded seven distinct ITS2 haplotypes. This polymorphism was extended to the $A$. sculptum sequences from GenBank, as they also represented distinct haplotypes in the phylogenetic tree (Fig. 2). The haplotypes of $A$. cajennense (s.s.) (haplotype A) and A. sculptum (haplotypes B-H) generated in the present study have been submitted to GenBank under the accession numbers KU169881-KU169888.

\section{Distribution maps}

A total of 58 localities with the occurrence of only $A$. cajennense (s.s.), 184 for only A. sculptum, 31 for both $A$. cajennense (s.s.) and A. sculptum, and 36 for A. cajennense (s.l.) (only male specimens) were used to construct distribution maps according to geopolitical regions (Fig. 3), biomes (Fig. 4), climate (Fig. 5) and natural vegetation cover (Fig. 6). The three field expeditions encompassed transition areas of the two species in Brazil, since almost all localities with sympatric occurrence of $A$. cajennense (s.s.) and A. sculptum were derived from these expeditions (Fig. 3). Therefore, these transition areas correspond primarily to the states of Maranhão and Tocantins in eastern Brazil, Mato Grosso in central Brazil, and Mato Grosso and Rondônia in western Brazil. These transition areas correspond to the geographic boundaries of the Amazon and Cerrado biomes (Fig. 4). In addition to these transition areas, $A$. cajennense (s.s.) was restricted to areas within the Amazon biome, whereas A. sculptum was found in the Cerrado, Pantanal, and Atlantic forest biomes. Interestingly, the single record of $A$. sculptum in the Caatinga biome (Fig. 4) was from an area of tropical climate, instead of 
Table 1 Data for the ticks Amblyomma cajennense (sensu stricto) [A.c.(s.s.)], Amblyomma sculptum (A.s.) and Amblyomma cajennense (sensu lato) [A.c.(s.l.)] collected during three field expeditions in Brazil ${ }^{a}$

\begin{tabular}{|c|c|c|c|c|c|c|c|}
\hline \multirow[t]{3}{*}{ Exp. } & \multicolumn{3}{|c|}{ Number of ticks } & \multirow[t]{3}{*}{ Host } & \multirow[t]{3}{*}{ Municipality } & \multirow[t]{3}{*}{ State } & \multirow{3}{*}{$\begin{array}{l}\text { Geographical } \\
\text { coordinates }(\mathrm{S}, \mathrm{W})\end{array}$} \\
\hline & A.c.(s.s.) & A.s. & A.c.(s.l.) & & & & \\
\hline & 우우 & 우우 & $\hat{\partial} \sigma^{\lambda}$ & & & & \\
\hline $1 s t$ & & & 1 & Equus caballus & Cach. do Piriá & Pará & $1^{\circ} 50.888^{\prime}, 45^{\circ} 23.452^{\prime}$ \\
\hline $1 s t$ & 21 & & 59 & E. caballus & S. G. Araguaia & Pará & $5^{\circ} 59.592^{\prime}, 48^{\circ} 37.947^{\prime}$ \\
\hline $1 s t$ & 1 & 1 & 1 & E. caballus & S. G. Araguaia & Pará & $6^{\circ} 06.594^{\prime}, 48^{\circ} 35.672^{\prime}$ \\
\hline $1 s t$ & 2 & 8 & 7 & E. caballus & Alvorada & Tocantins & $12^{\circ} 23.111^{\prime}, 49^{\circ} 07.061^{\prime}$ \\
\hline $1 s t$ & 3 & 3 & 6 & E. caballus & Araguaína & Tocantins & $7^{\circ} 19.570^{\prime}, 48^{\circ} 18.255^{\prime}$ \\
\hline 1 st & 7 & & 10 & E. caballus & Araguanã & Tocantins & $6^{\circ} 35.582^{\prime}, 48^{\circ} 38.493^{\prime}$ \\
\hline $1 s t$ & 3 & & & E. caballus & Araguanã & Tocantins & $6^{\circ} 51.336^{\prime}, 48^{\circ} 30.986^{\prime}$ \\
\hline $1 s t$ & & 12 & 25 & E. caballus & Araguatins & Tocantins & $5^{\circ} 45.278^{\prime}, 48^{\circ} 03.427^{\prime}$ \\
\hline $1 s t$ & & 1 & 4 & E. caballus & Barrolândia & Tocantins & $9^{\circ} 44.843^{\prime}, 48^{\circ} 42.105^{\prime}$ \\
\hline $1 s t$ & 1 & 10 & 12 & E. caballus & Barrolândia & Tocantins & $9^{\circ} 51.657^{\prime}, 48^{\circ} 43.446^{\prime}$ \\
\hline $1 s t$ & 1 & 1 & 2 & Free-living & Bras. Tocantins & Tocantins & $8^{\circ} 29.058^{\prime}, 48^{\circ} 29.054^{\prime}$ \\
\hline $1 s t$ & 1 & 2 & 3 & E. caballus & Brej. Nazaré & Tocantins & $11^{\circ} 02.660^{\prime}, 48^{\circ} 45.039^{\prime}$ \\
\hline $1 s t$ & & 1 & 19 & E. caballus & Brej. Nazaré & Tocantins & $10^{\circ} 59.359^{\prime}, 48^{\circ} 33.451^{\prime}$ \\
\hline $1 s t$ & 5 & 18 & 44 & E. caballus & Cariri Tocantins & Tocantins & $11^{\circ} 53.841^{\prime}, 49^{\circ} 10.020^{\prime}$ \\
\hline $1 s t$ & 1 & 6 & 7 & E. caballus & Crix. Tocantins & Tocantins & $11^{\circ} 12.863^{\prime}, 48^{\circ} 55.806^{\prime}$ \\
\hline $1 s t$ & 4 & 74 & 141 & E. caballus & Figueirópolis & Tocantins & $12^{\circ} 13.940^{\prime}, 49^{\circ} 09.919^{\prime}$ \\
\hline $1 s t$ & & 6 & 32 & Sus scrofa & Fort. Tabocão & Tocantins & $9^{\circ} 07.003^{\prime}, 48^{\circ} 32.006^{\prime}$ \\
\hline $1 s t$ & 2 & 18 & 17 & E. caballus & Guaraí & Tocantins & $8^{\circ} 58.520^{\prime}, 48^{\circ} 29.955^{\prime}$ \\
\hline 1 st & & 9 & 11 & E. caballus & Gurupi & Tocantins & $11^{\circ} 33.700^{\prime}, 49^{\circ} 01.503^{\prime}$ \\
\hline $1 s t$ & 5 & 9 & 19 & E. caballus & Gurupi & Tocantins & $11^{\circ} 49.618^{\prime}, 49^{\circ} 07.801^{\prime}$ \\
\hline $1 s t$ & & 6 & 11 & E. caballus & Luzinópolis & Tocantins & $6^{\circ} 10.583^{\prime}, 47^{\circ} 51.664^{\prime}$ \\
\hline $1 s t$ & 8 & 5 & 27 & Myrmecophaga tridactyla & Miranorte & Tocantins & $9^{\circ} 24.910^{\prime}, 48^{\circ} 34.253^{\prime}$ \\
\hline 1 st & & 14 & 16 & E. caballus & Nazaré & Tocantins & $6^{\circ} 23.002^{\prime}, 47^{\circ} 42.193^{\prime}$ \\
\hline $1 s t$ & 1 & & 3 & E. caballus & Nova Olinda & Tocantins & $7^{\circ} 47.974^{\prime}, 48^{\circ} 27.873^{\prime}$ \\
\hline $1 s t$ & 1 & 4 & 1 & E. caballus & N. Rosalândia & Tocantins & $10^{\circ} 34.032^{\prime}, 48^{\circ} 55.116^{\prime}$ \\
\hline $1 s t$ & & 3 & 1 & E. caballus & Par. Tocantins & Tocantins & $10^{\circ} 07.183^{\prime}, 48^{\circ} 52.998^{\prime}$ \\
\hline $1 s t$ & & 2 & 10 & E. caballus & Porto Nacional & Tocantins & $10^{\circ} 29.502^{\prime}, 48^{\circ} 20.153^{\prime}$ \\
\hline $1 s t$ & 7 & 4 & 45 & E. caballus & S. R. Tocantins & Tocantins & $10^{\circ} 54.404^{\prime}, 48^{\circ} 54.798^{\prime}$ \\
\hline $1 s t$ & 1 & 2 & 1 & E. caballus & S. B. Tocantins & Tocantins & $5^{\circ} 58.436^{\prime}, 47^{\circ} 52.737^{\prime}$ \\
\hline $1 s t$ & 1 & 1 & 24 & E. caballus & Talismã & Tocantins & $12^{\circ} 47.346^{\prime}, 49^{\circ} 05.470^{\prime}$ \\
\hline $1 s t$ & 2 & 35 & 27 & E. caballus & Tocantinópolis & Tocantins & $6^{\circ} 15.035^{\prime}, 47^{\circ} 27.741^{\prime}$ \\
\hline $1 s t$ & 1 & 3 & 6 & E. caballus & Tocantinópolis & Tocantins & $6^{\circ} 20.687^{\prime}, 47^{\circ} 33.018^{\prime}$ \\
\hline $1 s t$ & 1 & & & E. caballus & Xambioá & Tocantins & $6^{\circ} 24.783^{\prime}, 48^{\circ} 32.321^{\prime}$ \\
\hline $1 s t$ & & & 10 & E. caballus & Bequimão & Maranhão & $2^{\circ} 27.784^{\prime}, 44^{\circ} 47.416^{\prime}$ \\
\hline $1 s t$ & 7 & & 18 & S. scrofa & B. Jesus Selvas & Maranhão & $4^{\circ} 31.142^{\prime}, 46^{\circ} 46.691^{\prime}$ \\
\hline $1 s t$ & & & 2 & E. caballus & Imperatriz & Maranhão & $5^{\circ} 33.209^{\prime}, 47^{\circ} 27.766^{\prime}$ \\
\hline $1 s t$ & & & 3 & E. caballus & Pinheiro & Maranhão & $2^{\circ} 33.518^{\prime}, 59^{\circ} 47.764^{\prime}$ \\
\hline $1 s t$ & 2 & & 2 & E. caballus & Porto Franco & Maranhão & $6^{\circ} 20.177^{\prime}, 47^{\circ} 24.480^{\prime}$ \\
\hline 1 st & 1 & & 7 & E. caballus & Santa Helena & Maranhão & $2^{\circ} 15.681^{\prime}, 45^{\circ} 16.235^{\prime}$ \\
\hline $1 s t$ & & & 4 & E. caballus & Santa Inês & Maranhão & $3^{\circ} 41.666^{\prime}, 45^{\circ} 24.301^{\prime}$ \\
\hline $1 s t$ & 9 & & 7 & E. caballus & Santa Inês & Maranhão & $3^{\circ} 52.575^{\prime}, 45^{\circ} 32.757^{\prime}$ \\
\hline
\end{tabular}


Table 1 Data for the ticks Amblyomma cajennense (sensu stricto) [A.c.(s.s.)], Amblyomma sculptum (A.s.) and Amblyomma cajennense (sensu lato) [A.c.(s.l.)] collected during three field expeditions in Brazil ${ }^{\mathrm{a}}$ (Continued)

\begin{tabular}{|c|c|c|c|c|c|c|c|}
\hline $1 s t$ & 7 & & 5 & E. caballus & S. Luzia do Tide & Maranhão & $4^{\circ} 04.668^{\prime}, 45^{\circ} 57.052^{\prime}$ \\
\hline $1 s t$ & & 4 & 2 & E. caballus & Estrela Norte & Goiás & $13^{\circ} 49.376^{\prime}, 49^{\circ} 01.775^{\prime}$ \\
\hline $1 s t$ & 1 & 2 & 2 & E. caballus & Porangatu & Goiás & $12^{\circ} 52.722^{\prime}, 49^{\circ} 06.359^{\prime}$ \\
\hline $1 s t$ & & 5 & 5 & E. caballus & Porangatu & Goiás & $12^{\circ} 53.209^{\prime}, 49^{\circ} 06.390^{\prime}$ \\
\hline $1 s t$ & & 6 & 21 & E. caballus & Porangatu & Goiás & $13^{\circ} 24.868^{\prime}, 49^{\circ} 08.118^{\prime}$ \\
\hline $1 s t$ & & 1 & 4 & E. caballus & Porangatu & Goiás & $13^{\circ} 15.364^{\prime}, 49^{\circ} 08.122^{\prime}$ \\
\hline $1 s t$ & & 7 & 18 & E. caballus & S. Ter. Goiás & Goiás & $13^{\circ} 39.022^{\prime}, 49^{\circ} 02.094^{\prime}$ \\
\hline 2nd & 5 & 12 & 7 & E. caballus & Pimenta Bueno & Rondônia & $11^{\circ} 52.328^{\prime}, 60^{\circ} 59.868^{\prime}$ \\
\hline 2nd & 2 & & 1 & E. caballus & Presid. Médici & Rondônia & $11^{\circ} 09.754^{\prime}, 61^{\circ} 54.095^{\prime}$ \\
\hline 2nd & 2 & & & Free-living & Vilhena & Rondônia & $12^{\circ} 43.412^{\prime}, 60^{\circ} 15.417^{\prime}$ \\
\hline 2nd & 1 & & 4 & Free-living & Vilhena & Rondônia & $12^{\circ} 29.545^{\prime}, 60^{\circ} 28.309^{\prime}$ \\
\hline 2nd & 5 & & 6 & E. caballus & Comodoro & Mato Grosso & $13^{\circ} 56.276^{\prime}, 59^{\circ} 45.634^{\prime}$ \\
\hline 2nd & 32 & & 7 & Free-living & Comodoro & Mato Grosso & $13^{\circ} 56.276^{\prime}, 59^{\circ} 45.634^{\prime}$ \\
\hline 2nd & 2 & & & Free-living & Comodoro & Mato Grosso & $13^{\circ} 19.058^{\prime}, 59^{\circ} 52.654^{\prime}$ \\
\hline 2nd & & & 1 & Free-living & Comodoro & Mato Grosso & $13^{\circ} 05.216^{\prime}, 59^{\circ} 53.509^{\prime}$ \\
\hline 2nd & 4 & & 3 & Free-living & Comodoro & Mato Grosso & $13^{\circ} 00.277^{\prime}, 59^{\circ} 57.472^{\prime}$ \\
\hline 2nd & 1 & 49 & 26 & E. caballus & Conq. d'Oeste & Mato Grosso & $14^{\circ} 46.905^{\prime}, 59^{\circ} 20.774^{\prime}$ \\
\hline 2nd & 4 & & 6 & E. caballus & Nova Lacerda & Mato Grosso & $14^{\circ} 10.232^{\prime}, 59^{\circ} 42.056^{\prime}$ \\
\hline 2nd & 1 & & 1 & Free-living & Nova Lacerda & Mato Grosso & $14^{\circ} 07.560^{\prime}, 59^{\circ} 42.607^{\prime}$ \\
\hline 2nd & & & 1 & E. caballus & Pont. Lacerda & Mato Grosso & $15^{\circ} 09.292^{\prime}, 59^{\circ} 28.354^{\prime}$ \\
\hline 2nd & & 4 & 3 & E. caballus & Pont. Lacerda & Mato Grosso & $15^{\circ} 20.685^{\prime}, 59^{\circ} 23.777^{\prime}$ \\
\hline 2nd & & 3 & & E. caballus & Pont. Lacerda & Mato Grosso & $14^{\circ} 53.271^{\prime}, 59^{\circ} 16.735^{\prime}$ \\
\hline 2nd & & 3 & 3 & E. caballus & V. B. S. Trindade & Mato Grosso & $15^{\circ} 07.393^{\prime}, 59^{\circ} 34.550^{\prime}$ \\
\hline 2nd & & 1 & 1 & E. caballus & V. B. S. Trindade & Mato Grosso & $15^{\circ} 00.374^{\prime}, 59^{\circ} 57.109^{\prime}$ \\
\hline 2nd & & 5 & 11 & E. caballus & V. B. S. Trindade & Mato Grosso & $14^{\circ} 58.490^{\prime}, 59^{\circ} 59.009^{\prime}$ \\
\hline 2nd & 1 & 31 & 31 & Free-living & V. B. S. Trindade & Mato Grosso & $14^{\circ} 55.576^{\prime}, 60^{\circ} 01.216^{\prime}$ \\
\hline 2nd & & 11 & 11 & Free-living & V. B. S. Trindade & Mato Grosso & $15^{\circ} 05.634^{\prime}, 59^{\circ} 51.829^{\prime}$ \\
\hline $3 r d$ & & 10 & 34 & E. caballus & Diamantino & Mato Grosso & $14^{\circ} 33.153^{\prime}, 56^{\circ} 13.418^{\prime}$ \\
\hline $3 r d$ & & 2 & & E. caballus & Jangada & Mato Grosso & $15^{\circ} 48.708^{\prime}, 56^{\circ} 39.218^{\prime}$ \\
\hline $3 r d$ & 3 & 1 & 7 & E. caballus & Lucas Rio Verde & Mato Grosso & $13^{\circ} 45.656^{\prime}, 55^{\circ} 52.487^{\prime}$ \\
\hline $3 r d$ & & 8 & 9 & E. caballus & Nobres & Mato Grosso & $14^{\circ} 43.526^{\prime}, 56^{\circ} 19.525^{\prime}$ \\
\hline $3 r d$ & & 8 & 3 & E. caballus & Rosário Oeste & Mato Grosso & $14^{\circ} 48.500^{\prime}, 56^{\circ} 25.580^{\prime}$ \\
\hline $3 r d$ & & 12 & 11 & E. caballus & Rosário Oeste & Mato Grosso & $14^{\circ} 48.408^{\prime}, 56^{\circ} 25.278^{\prime}$ \\
\hline $3 r d$ & & & 21 & E. caballus & Sinop & Mato Grosso & $11^{\circ} 52.466^{\prime}, 55^{\circ} 36.316^{\prime}$ \\
\hline $3 r d$ & 11 & & 7 & E. caballus & Sinop & Mato Grosso & $11^{\circ} 55.516^{\prime}, 55^{\circ} 42.000^{\prime}$ \\
\hline $3 r d$ & 68 & & 137 & Tapirus terrestris & Sinop & Mato Grosso & $13^{\circ} 45.660^{\prime}, 55^{\circ} 52.487^{\prime}$ \\
\hline Total & 249 & 443 & 1,054 & & & & \\
\hline
\end{tabular}

Exp.: Field expedition

a 1st expedition: considered to represent sampling in the eastern transition area of $A$. cajennense (s.s.) and A. sculptum. 2nd expedition: considered to represent sampling in the western transition area of the two tick species in Brazil. 3rd expedition: considered to represent the central transition area of the two species in Brazil 
Table 2 Data for the ticks Amblyomma cajennense (sensu stricto) (s.s.) and Amblyomma sculptum from Brazil used for molecular and phylogenetic analyses in the present study

\begin{tabular}{|c|c|c|c|c|c|c|c|c|}
\hline \multirow[t]{2}{*}{ Tick species } & \multirow[t]{2}{*}{ Gender } & \multirow[t]{2}{*}{ Host } & \multirow[t]{2}{*}{ Date } & \multicolumn{3}{|l|}{ Locality } & \multirow{2}{*}{$\begin{array}{l}\text { No. of } \\
\text { specimens }\end{array}$} & \multirow{2}{*}{$\begin{array}{l}\text { Haplotype } \\
\text { code }\end{array}$} \\
\hline & & & & Municipality & State & $\begin{array}{l}\text { Geographical } \\
\text { Coordinates }(S, W)\end{array}$ & & \\
\hline A. cajennense (s.s.) & $\pi$ & Equus caballus & Jan/2012 & Cachoeira do Piriá & Pará & $1^{\circ} 50^{\prime}, 45^{\circ} 23^{\prime}$ & 1 & $A$ \\
\hline A. cajennense (s.s.) & $\hat{0}$ & E. caballus & Oct/2011 & S. Dom. Capim & Pará & $1^{\circ} 40^{\prime}, 47^{\circ} 46^{\prime}$ & 1 & $A$ \\
\hline A. cajennense (s.s.) & 우 & E. caballus & Jan/2012 & S. Ger. Araguaia & Pará & $6^{\circ} 6^{\prime}, 48^{\circ} 35^{\prime}$ & 2 & $A$ \\
\hline A. cajennense (s.s.) & $\hat{0}$ & Bubalus bubalis & Sep/2011 & S. Franc. Guaporé & Rondônia & $12^{\circ} 3^{\prime}, 63^{\circ} 34^{\prime}$ & 2 & $A$ \\
\hline A. cajennense (s.s.) & q & Free-living & Mar/2002 & Vilhena & Rondônia & $12^{\circ} 44^{\prime}, 60^{\circ} 8^{\prime}$ & 3 & $A$ \\
\hline A. cajennense (s.s.) & $q^{a}$ & Canis familiaris & Aug/2011 & Cristalândia & Tocantins & $10^{\circ} 36^{\prime}, 49^{\circ} 11^{\prime}$ & 2 & $A$ \\
\hline A. cajennense (s.s.) & $\hat{\sigma}$ & E. caballus & $\operatorname{Jan} / 2012$ & Bequimão & Maranhão & $2^{\circ} 27^{\prime}, 44^{\circ} 47^{\prime}$ & 2 & $A$ \\
\hline A. cajennense (s.s.) & $\hat{0}$ & E. caballus & Jan/2012 & Chapadinha & Maranhão & $3^{\circ} 44^{\prime}, 43^{\circ} 21^{\prime}$ & 2 & $A$ \\
\hline A. cajennense (s.s.) & $\hat{\sigma}$ & E. caballus & Jan/2012 & Pinheiro & Maranhão & $2^{\circ} 33^{\prime}, 59^{\circ} 47^{\prime}$ & 2 & $A$ \\
\hline A. cajennense (s.s.) & $\hat{\sigma}$ & E. caballus & Jan/2012 & Santa Inês & Maranhão & $3^{\circ} 41^{\prime}, 45^{\circ} 24^{\prime}$ & 2 & $A$ \\
\hline A. cajennense (s.s.) & $\hat{\sigma}$ & Free-living & Jan/2012 & Comodoro & Mato Grosso & $13^{\circ} 5^{\prime}, 59^{\circ} 53^{\prime}$ & 1 & A \\
\hline A. cajennense (s.s.) & $\hat{o}$ & E. caballus & Apr/2011 & Confresa & Mato Grosso & $10^{\circ} 38^{\prime}, 51^{\circ} 34^{\prime}$ & 2 & $A$ \\
\hline A. cajennense (s.s.) & $\hat{o}$ & E. caballus & Jan/2012 & Pontes e Lacerda & Mato Grosso & $15^{\circ} 9^{\prime}, 59^{\circ} 28^{\prime}$ & 1 & $A$ \\
\hline A. cajennense (s.s.) & $\hat{o}$ & E. caballus & Feb/2013 & Sinop & Mato Grosso & $11^{\circ} 52^{\prime}, 55^{\circ} 36^{\prime}$ & 1 & $A$ \\
\hline A. sculptum & 우 & E. caballus & Jan/2012 & Pimenta Bueno & Rondônia & $11^{\circ} 52^{\prime}, 60^{\circ} 59^{\prime}$ & 2 & $B, C$ \\
\hline A. sculptum & $\hat{0}$ & Free-living & Jun/2011 & Tocantinópolis & Tocantins & $6^{\circ} 19^{\prime}, 47^{\circ} 24^{\prime}$ & 2 & $\mathrm{D}$ \\
\hline A. sculptum & $\hat{\sigma}$ & E. caballus & Aug/2012 & Campo Formoso & Bahia & $10^{\circ} 30^{\prime}, 40^{\circ} 19^{\prime}$ & 1 & $E$ \\
\hline A. sculptum & $\hat{o}$ & E. caballus & Jul/2011 & Balsas & Maranhão & $7^{\circ} 31^{\prime}, 46^{\circ} 2^{\prime}$ & 2 & $E, F$ \\
\hline A. sculptum & $\hat{0}$ & E. caballus & Jan/2012 & Imperatriz & Maranhão & $5^{\circ} 33^{\prime}, 47^{\circ} 27^{\prime}$ & 1 & G \\
\hline A. sculptum & $\hat{o}$ & Equus asinus & Jan/2011 & José de Freitas & Piauí & $4^{\circ} 45^{\prime}, 42^{\circ} 34^{\prime}$ & 2 & E \\
\hline A. sculptum & $\hat{\sigma}$ & E. caballus & Jun/2011 & Cumari & Goiás & $18^{\circ} 15^{\prime}, 48^{\circ} 9^{\prime}$ & 1 & E \\
\hline A. sculptum & 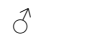 & E. caballus & Jan/2012 & Porangatu & Goiás & $12^{\circ} 52^{\prime}, 49^{\circ} 6^{\prime}$ & 1 & $E$ \\
\hline A. sculptum & $\hat{0}$ & E. caballus & Jul/2011 & Poconé & Mato Grosso & $16^{\circ} 15^{\prime}, 56^{\circ} 37^{\prime}$ & 1 & $\mathrm{H}$ \\
\hline A. sculptum & 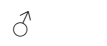 & Sus scrofa & Aug/2010 & Corumbá & M. Grosso do Sul & $19^{\circ} 0^{\prime}, 57^{\circ} 39^{\prime}$ & 2 & $E$ \\
\hline A. sculptum & $\hat{\sigma}$ & E. caballus & $\mathrm{Jul} / 2011$ & Pinheiros & Espírito Santo & $18^{\circ} 24^{\prime}, 40^{\circ} 13^{\prime}$ & 1 & E \\
\hline A. sculptum & $\hat{\sigma}$ & E. caballus & Jan/2011 & Itabira & Minas Gerais & $19^{\circ} 37^{\prime}, 43^{\circ} 13^{\prime}$ & 2 & $E$ \\
\hline A. sculptum & $\hat{o}$ & E. caballus & May/2011 & Seropédica & Rio de Janeiro & $22^{\circ} 44^{\prime}, 43^{\circ} 42^{\prime}$ & 2 & E \\
\hline A. sculptum & $\hat{o}$ & Free-living & Oct/2011 & Americana & São Paulo & $22^{\circ} 44^{\prime}, 47^{\circ} 19^{\prime}$ & 2 & $E$ \\
\hline A. sculptum & $\hat{0}$ & E. caballus & Dec/2008 & Pirassununga & São Paulo & $21^{\circ} 59^{\prime}, 47^{\circ} 25^{\prime}$ & 3 & E \\
\hline A. sculptum & $\delta$ & E. caballus & May/2012 & Alvorada do Sul & Paraná & $22^{\circ} 46^{\prime}, 51^{\circ} 13^{\prime}$ & 1 & E \\
\hline Total & & & & & & & 50 & \\
\hline
\end{tabular}

${ }^{a}$ This tick was collected as an engorged nymph, and molted to the adult stage in the laboratory

the dominant semiarid climate of this biome (Fig. 5). The distribution of $A$. cajennense (s.s.) was almost restricted to areas with equatorial climate (which generally coincides with Amazon biome), whereas A. sculptum was almost restricted to areas with tropical climate (which generally coincides with Cerrado and Atlantic forest biomes) (Fig. 5). There were very few records of A. sculptum or A. cajennense (s.l.) from areas with subtropical climate in southern Brazil. In addition, the
Pampa biome (southernmost Brazil) was the only biome where A. cajennense (s.l.) was absent in Brazil (Fig. 4). In the Amazon biome, the occurrence of $A$. cajennense (s.s.) was generally restricted to areas where the natural vegetation cover was absent. The same applies to $A$. sculptum in the Atlantic forest biome. On the other hand, in the Cerrado and Pantanal biomes, A. sculptum was found in areas with or without natural vegetation cover (Fig. 6). 


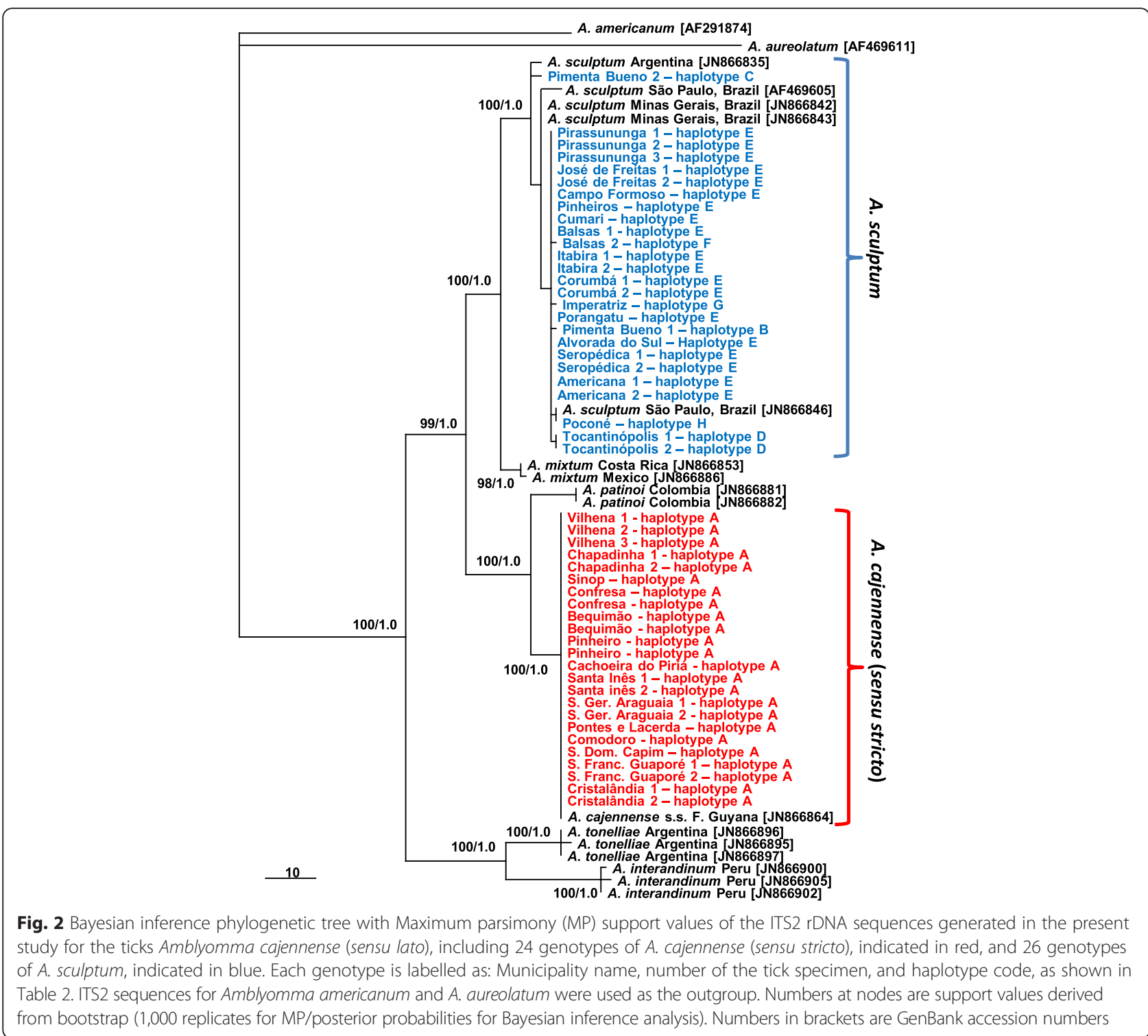

\section{Description of the nymph of $A$. cajennense (s.s.)}

[Based on 10 specimens; (Fig. 7).] Idiosoma. Length from apices of scapula to posterior body margin: 1,280-1,579 (1,444); maximum breadth 1,040-1,257 $(1,156)$; outline oval, with 11 festoons without tubercles. Scutum 681-784 (730) long, 835-944 (887) wide, breadth/length ratio 1.146-1.265 (1.214), inornate; large and deep punctations evenly distributed. Eyes not orbited at lateral scutal angles at level of scutal midlength. Cervical grooves deep in scutal anterior third, followed by rugose shallow depression in scutal median third. Spiracular plate triangular with rounded angles, with an evident dorsal prolongation, 256-317 (276) long, 192-257 (218) wide.
Gnathosoma. Length from palpal apices to dorsal posterior margin 373-473 (414), breadth 317-353 (331). Basis capituli rectangular, posterior margin slightly concave, without cornua; posterior margin convex ventrally, without auriculae. Palpi length 280-345 (307), article I with vestigial ventral prolongation, article II 165-208 (182) long, article III 86-116 (98) long. Hypostome rounded apically, 291-364 (316) long; length of toothed portion 164-204 (178); dentition $2 / 2$ with 7-8 teeth per row.

Legs. Coxa I with 2 pointed spurs separated by space equal or greater than breadth of external spur base; external spur about $2 \times$ longer than internal; coxae II-IV with small triangular spur. Trochanters without spur; 


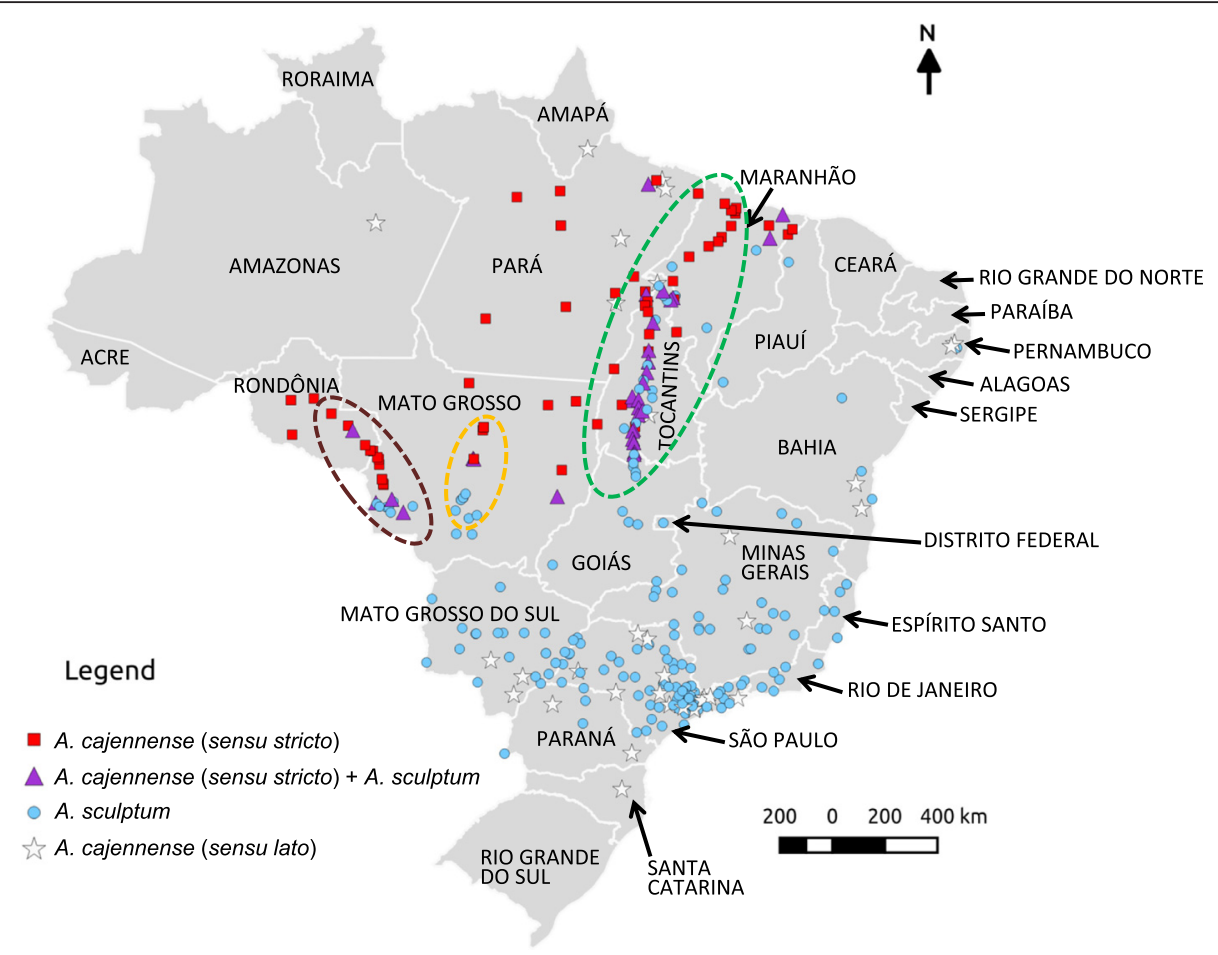

Fig. 3 Geopolitical map of Brazil, showing the localities of Amblyomma cajennense (sensu stricto), A. sculptum and A. cajennense (sensu lato) identified in the present study. Dashed line ellipses include the localities that were sampled during three field expeditions for tick collections, the first expedition in green, the second in brown and the third in orange

tarsus I 431-509 (450) long, 99-115 (109) wide; tarsus IV 320-372 (337) long, 72-99 (86) wide.

Remark: The nymph of $A$. cajennense (s.s.) is morphologically very similar to the nymph of $A$. sculptum, recently described [7]. Comparing the two descriptions, the only concrete difference is the scutal width/length ratio $[<1.3$ in A. cajennense (s.s.) $v s>1.3$ in $A$. sculptum]. Amblyomma cajennense (s.s.) can be separated from $A$. tonelliae by using the same criteria recently proposed to separate $A$. tonelliae from A. sculptum, based on scutum surface and the length of the anterolateral seta on coxa I [8].

\section{Discussion}

In the present study, examination of all lots of $A$. cajennense (s.l.) from two large tick collections of Brazil, plus collection of new material during three field expeditions, confirmed that A. cajennense (s.l.) is currently represented in Brazil by only two species, A. cajennense (s.s.) and A. sculptum. This result was corroborated by molecular analyses inferred from the ITS2 ribosomal gene. We chose this molecular marker because a recent study revealed that the ITS2 gene fragment is suitable for evolutionary studies on $A$. cajennense [5]. If any other species of A. cajennense (s.l.) occurs in Brazil, it might have a much more restricted distribution, since our analyses encompassed areas throughout the Brazilian land.

As previously reported [2, 5], our study confirms that A. cajennense (s.s.) occurs generally in the Amazon biome; however, almost all records were from the edge of this biome, among the so called "Seasonally Dry Tropical Forest" (SDTF) part of the Amazon biome; i.e., this tick is not found in thick of the rain forest [5], which generally means the inner part of the forest. While the absence of records from areas deeper inside the Amazon biome could be related to poor sampling in these less accessible areas, it is noteworthy that an extensive study in the state of Rondônia (Brazilian western Amazon) made multiple samplings throughout the state, and concluded that $A$. cajennense was generally absent from areas with dense Amazon forest [21]. The above statements are corroborated by our results shown in Fig. 6, where all records of $A$. cajennense (s.s.) are within areas with degraded forest cover, i.e. this species was not found at any area where natural vegetation cover remains in the Amazon biome. This geographic distribution could be related to particular abiotic conditions required by $A$. cajennense (s.s.), suggesting that this tick might not be adapted to extremely humid rainforests. On the other hand, the absence of $A$. cajennense (s.s.) 


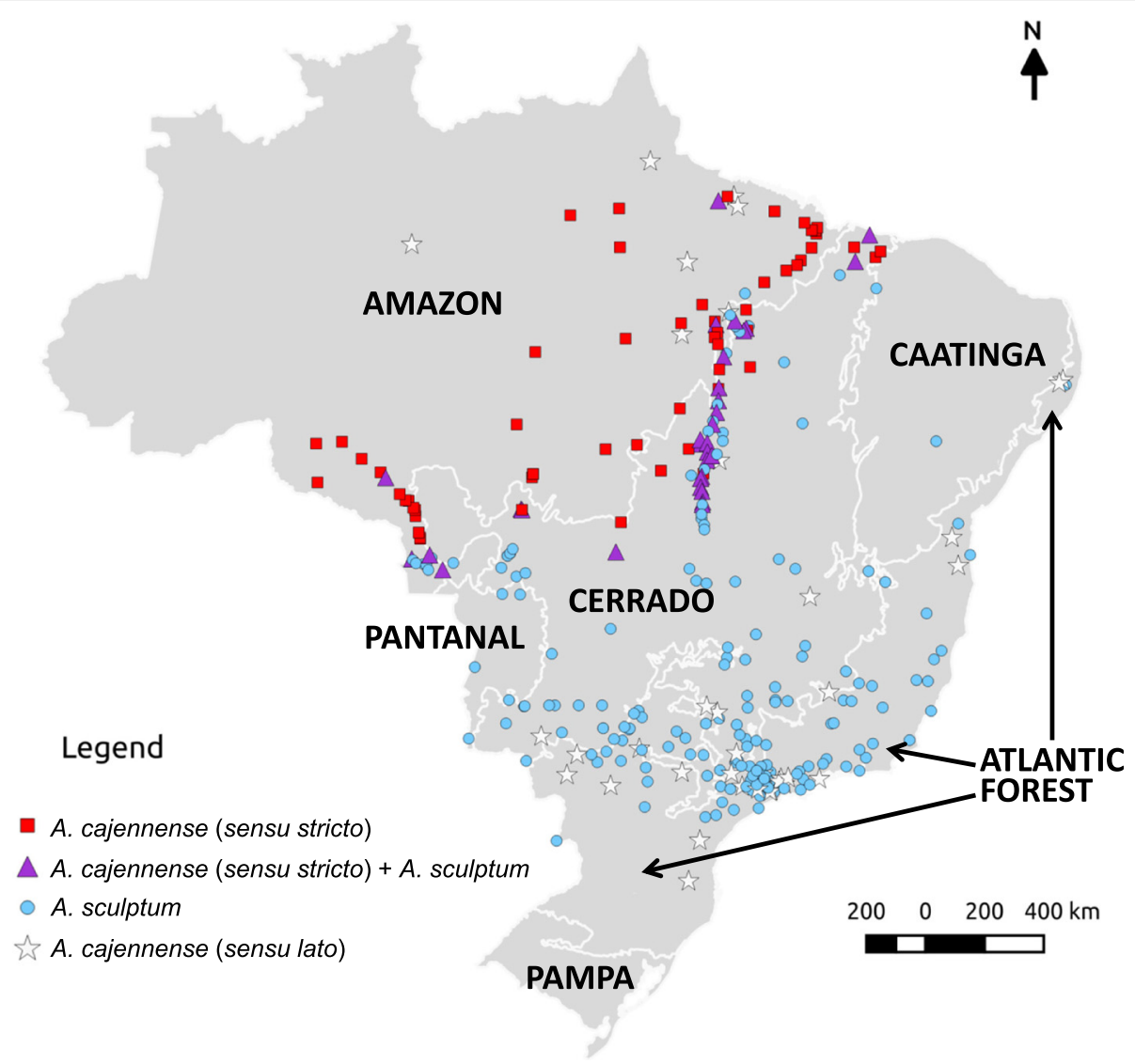

Fig. 4 Map of Brazil showing the localities of the ticks Amblyomma cajennense (sensu stricto), A. sculptum and A. cajennense (sensu lato) identified in the present study, according to the six major biomes (Amazon, Atlantic forest, Caatinga, Cerrado, Pantanal, Pampa) that compose the Brazilian land

deeper in the Cerrado biome (Brazilian savannah) could be related to unsuitable abiotic conditions at the opposite extreme, namely, the much drier seasonally drought period, typical for the tropical climate that prevails in the Cerrado (Figs. 3, 4).

The distribution of A. sculptum in Brazil generally encompassed entire Cerrado and Pantanal biomes and great part of the Atlantic forest biome (Fig. 3). This distribution coincides with the tropical climate. Interestingly, the absence of A. sculptum in southern Brazil (Fig. 2) coincides with the subtropical climate (Fig. 4), suggesting that the cooler temperatures during autumn/ winter in this region might be the limiting factor for the establishment of $A$. sculptum. This assumption is corroborated by previous modelling studies [1, 22]. While A. sculptum seems to be very adapted to the Cerrado biome, the same cannot be applied to the Caatinga biome, where the semiarid climate (Figs. 2, 3) is possibly the main limiting factor. Interestingly, our single record of $A$. sculptum in the Caatinga biome was from the state of Bahia (Figs. 2, 3), in an area that represents a narrow "invagination" of the tropical climate into the Caatinga (Fig. 4).

It is noteworthy that nearly all records of $A$. sculptum in the Atlantic forest biome were from areas where the natural vegetation cover has been degraded (Fig. 5), i.e. this tick was not found at any area where natural vegetation cover remains in the Atlantic forest biome. This result is corroborated by an extensive field study in a large Atlantic forest Reserve in Southern São Paulo, where $A$. sculptum (reported as A. cajennense) was never found inside the dense forest, where suitable hosts (e.g. tapirs) were abundant; on the other hand, A. sculptum was abundant in an open, degraded area within the Reserve [23]. The authors suggested that the distribution of $A$. sculptum has increased as a result of destruction of the Atlantic rainforest over the last few centuries. In fact, the Atlantic forest biome is indeed the most degraded Brazilian biome, which retains $<10 \%$ of its original natural vegetation cover [24]. This condition can be clearly observed in Figs. 2 and 5. It seems likely that abiotic factors of these degraded areas of the Atlantic forest 


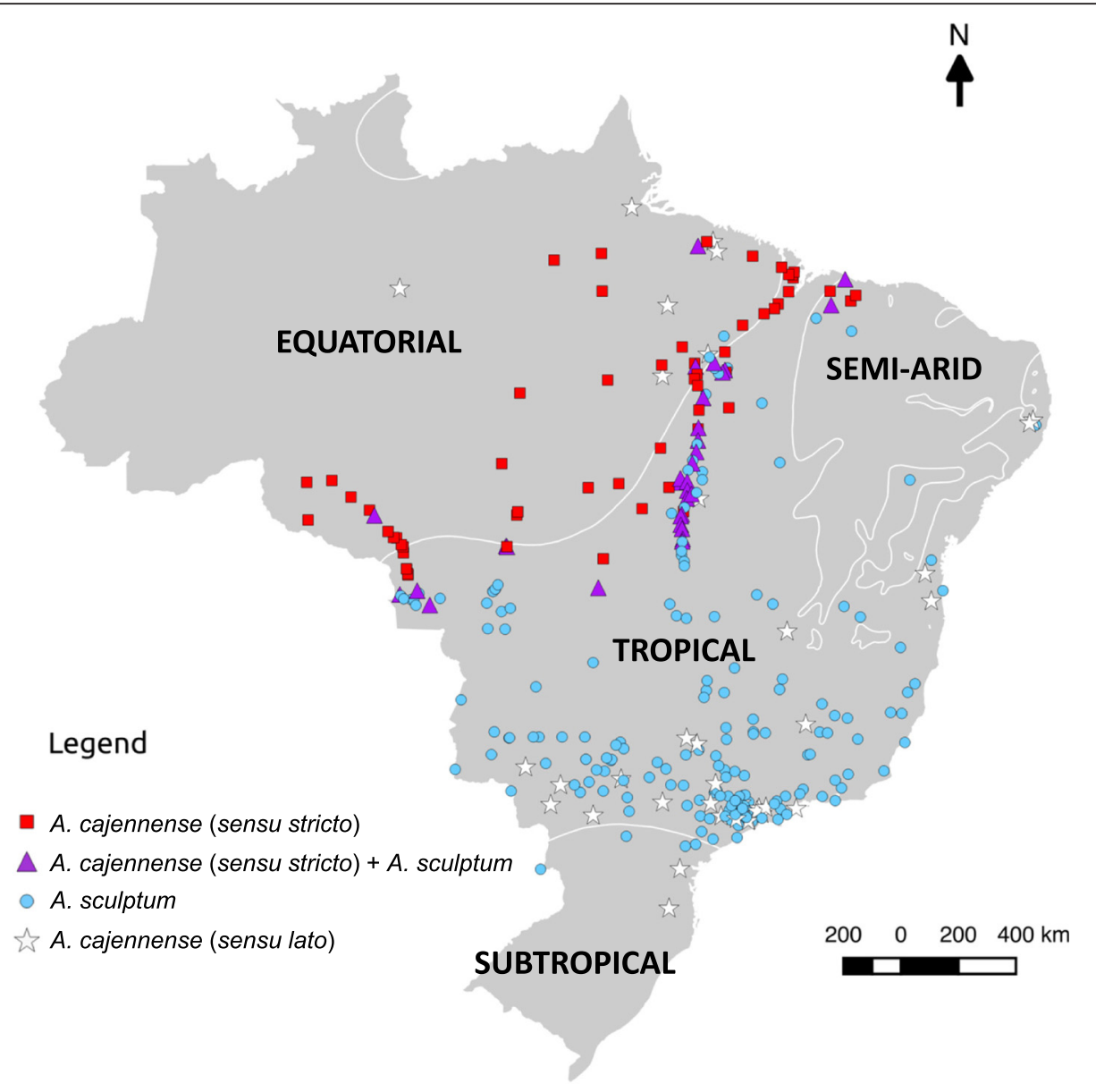

Fig. 5 Map of Brazil showing the localities of the ticks Amblyomma cajennense (sensu stricto), A. sculptum and A. cajennense (sensu lato) identified in the present study, according to the four major climates (Equatorial, Tropical, Semi-arid, and Subtropical) that occur in the Brazilian land

"simulate" the conditions of the Cerrado biome, where A. sculptum prevails on either natural or degraded areas, as shown in Fig. 5. This statement is corroborated by a previous study with the use of satellite imagery and the distribution of $A$. cajennense (s.l.) [1], which showed that both the Cerrado and the degraded Atlantic forest areas of Brazil presented similar normalised derived vegetation index (NVDI). This index is an indirect quantification of abiotic factors.

In the present study, we were able to find three transition areas of the distribution of A. cajennense (s.s.) and A. sculptum in Brazil, where these tick species were sympatric (Fig. 2). These results are in agreement with a recent environmental modelling study suggesting that $A$. cajennense (s.s.) and A. sculptum may overlap in parts of their range due to some similarities in abiotic variables [22]. Because these transition areas corresponded to the boundaries between the Amazon and Cerrado biome, or between the equatorial and tropical climate, we can infer that these transition areas represent extreme abiotic conditions for the distribution of the two species.

Continuing degradation of the Amazon forest, with replacement of the original forest cover by Cerrado-like vegetation cover may favour the expansion of $A$. sculptum into these areas. A potential example of this condition is the state of Rondônia, where Labruna et al. [21] did not find any A. sculptum during a large, extensive tick survey throughout the state during 2000-2005. Nearly one decade later, in 2012 (during our second field expedition), we were able to find an established population of A. sculptum infesting horses in Pimenta Bueno municipality (corresponds to the single record of $A$. sculptum in Rondônia in Fig. 2). Because Rondônia is one of the Brazilian states with the highest deforestation and forest degradation indices during the last two decades [25], this condition might have facilitated the expansion of A. sculptum into the state. This scenario has extreme medical relevance, since in Brazil A. sculptum is 


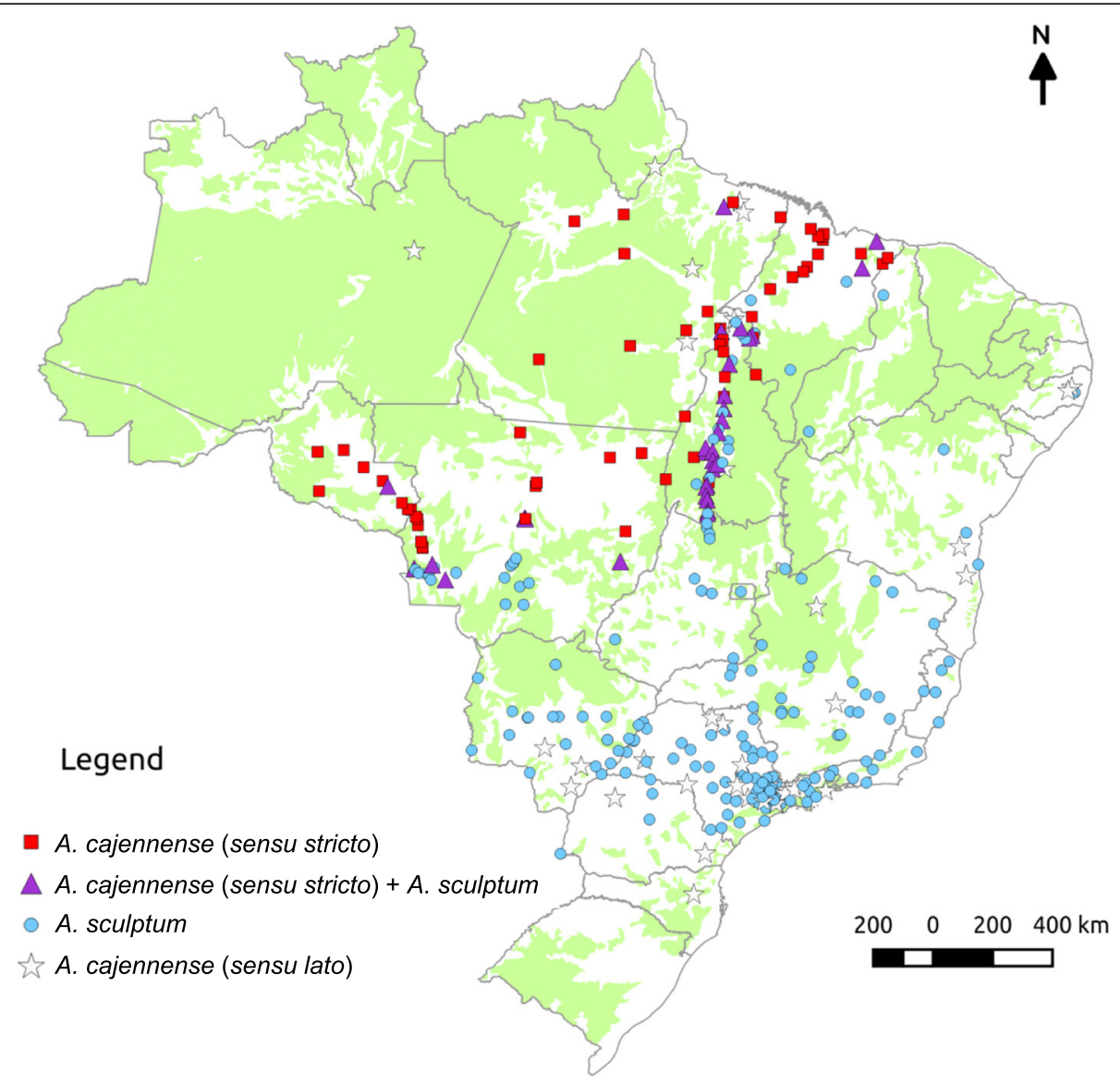

Fig. 6 Map of Brazil showing the localities of the ticks Amblyomma cajennense (sensu stricto), A. sculptum, and A. cajennense (sensu lato) identified in the present study, according to natural vegetation cover, represented in green

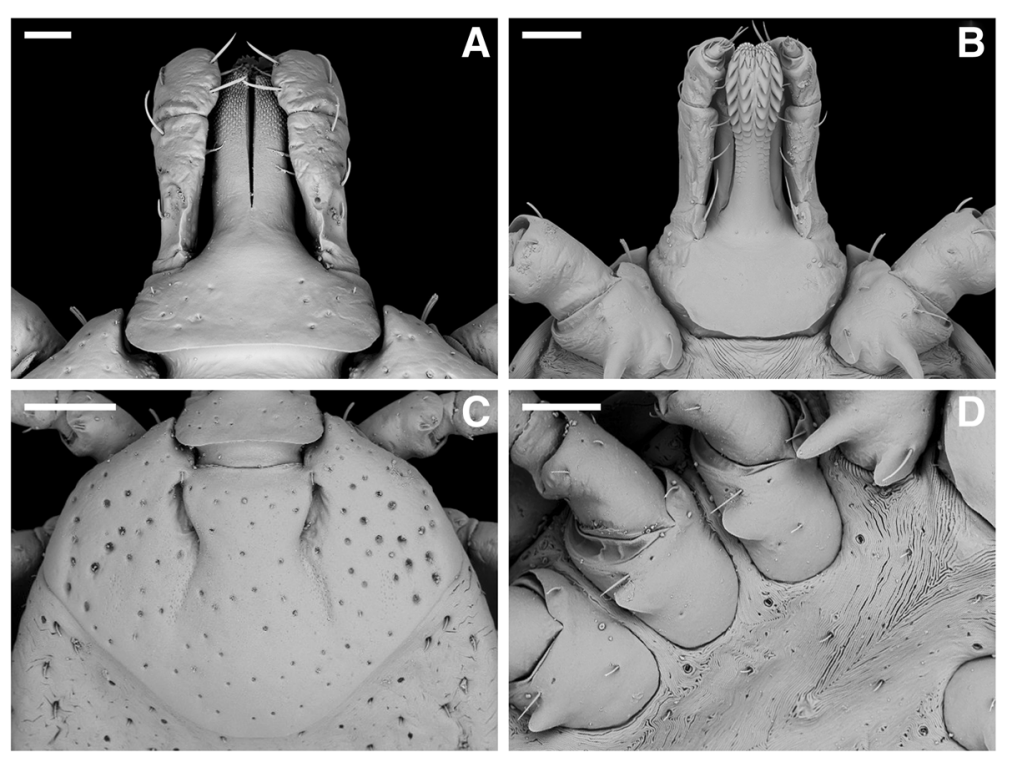

Fig. 7 Amblyomma cajennense (sensu stricto) nymph. a Dorsal gnathosoma (capitulum); b Ventral gnathosoma; c Scutum; d Coxae I-IV. Scale-bars: a, $60 \mu \mathrm{m} ; \mathrm{b}, \mathrm{d}, 100 \mu \mathrm{m} ; \mathrm{c}, 200 \mu \mathrm{m}$ 
the most important vector of the bacterium Rickettsia rickettsii, the etiological agent of the Brazilian spotted fever, the most lethal spotted fever of the world [26]. On the other hand, $A$. cajennense (s.s.) has only been found infected by Rickettsia amblyommii, a much less or nonpathogenic rickettsial agent [27]. Hence, expansion of $A$. sculptum into northern Brazil could involve expansion of other tick-borne diseases, especially because A. sculptum is indeed the most common human-biting tick in Brazil [28]. Our data corroborate this assumption, since $H$. sapiens was the most frequent host species for $A$. sculptum among the two tick collections examined in the present study.

\section{Conclusions}

Results of the present study confirmed that A. cajennense (s.l.) is currently represented in Brazil by only two species, A. cajennense (s.s.) and A. sculptum. While the two species have distinct distribution areas in the country, they are found in sympatry in some transition areas. The current distribution of $A$. cajennense (s.l.) has important implications to public health, since in Brazil $A$. sculptum is the most important vector of the bacterium $R$. rickettsii, the etiological agent of the most lethal spotted fever of the world, the Brazilian spotted fever.

\section{Additional file}

Additional file 1: Table S1. Records of Amblyomma cajennense sensu stricto (s.s.), Amblyomma sculptum, and Amblyomma cajennense sensu lato (s.l.) that were examined in the tick collections "Acari Colletion of the Instituto Butantan" (IBSP), São Paulo, Brazil, and "Coleção Nacional de Carrapatos" (CNC) of the University of São Paulo, São Paulo, Brazil. (PDF $1091 \mathrm{~kb}$ )

\section{Competing interests}

The authors declare that they have no competing interests.

\section{Authors' contributions}

TFM, FBC, FAT, LMAC, CRLP, RCP, AS, AKC, MCH, and MBL conducted the field studies, and revised the manuscript. TFM, ARMB, HRB, AM, and MBL performed the molecular and phylogenetic analyses. RAD and AGAG constructed the distribution maps. TFM, PHN and MBL described the nymph. TFM, DGR, DMB-B, and MBL revised the tick collections. All authors read and approved the final manuscript.

\section{Acknowledgements}

Thanks to Gustavo Fernochi for his technical support in editing photographs. This work was supported by Conselho Nacional de Desenvolvimento Científico e Tecnológico (CNPq, Project 141945/2010-9, Fundação de Amparo à Pesquisa do Estado de São Paulo (FAPESP, Project 2014/14464-1), and Coordenação de Aperfeiçoamento de Pessoal de Nível Superior (CAPES/ PROEX 2327/2015), Brazil.

\section{Author details}

${ }^{1}$ Departamento de Medicina Veterinária Preventiva e Saúde Animal, Faculdade de Medicina Veterinária e Zootecnia, Universidade de São Paulo, Av. Prof. Orlando Marques de Paiva 87, São Paulo 05508-270, Brazil. ${ }^{2}$ Faculdade São Lucas, R. Alexandre Guimarães Areal 1927, Porto Velho, Rondônia 78916-450, Brazil. ²Departamento de Parasitologia, Instituto de Ciências Biomédicas, Universidade de São Paulo, Av. Professor Lineu Prestes
1374, São Paulo 05389-970, Brazil. ${ }^{4}$ Coordenação Geral do Programa Nacional de Controle da Malária, Setor Comercial Sul, Quadra 04, Bloco A, Edifício Principal, $6^{\circ}$ Andar, Brasília, Distrito Federal 70304-000, Brazil. ${ }^{5}$ Departamento de Ciências Básicas e Produção Animal, Faculdade de Agronomia, Medicina Veterinária e Zootecnia, Universidade Federal de Mato Grosso, Av. Fernando Corrêa da Costa 2367, Cuiabá, Mato Grosso 78060-900, Brazil. ' Instituto Latino-Americano de Ciências da Vida e da Natureza, Universidade Federal da Integração Latino-Americana, Av. Tarquínio Joslin dos Santos 1000, Foz do I guaçu, Paraná 85870-901, Brazil. Universidade de Santo Amaro, R. Prof. Enéas de Siqueira Neto 340, São Paulo 04829-300, Brazil. ${ }^{8}$ Curso de Medicina Veterinária, Faculdade de Medicina Veterinária, Universidade Federal do Pará, Av. Maximino Porpino 1000, Castanhal, Pará 68740-000, Brazil. ${ }^{9}$ Deparatamento de Veterinária, Universidade Federal de Viçosa, Av. PH Rolfs, s/n, Viçosa, Minas Gerais 36570-000, Brazil. ${ }^{10}$ Universidade Federal do Vale do São Francisco, Campus de Ciências Agrárias, Rodovia BR 407, Km 12, Lote 543 - Projeto de Irrigação Senador Nilo Coelho, s/n, Petrolina, Pernambuco 56300-990, Brazil. ${ }^{11}$ Laboratório Especial de Coleções Zoológicas, Instituto Butantan, Av. Vital Brasil 1500, São Paulo 05503-900, Brazil.

Received: 11 January 2016 Accepted: 15 March 2016

Published online: 31 March 2016

\section{References}

1. Estrada-Peña A, Guglielmone AA, Mangold AJ. The distribution and ecological preferences of the tick Amblyomma cajennense (Acari: Ixodidae), an ectoparasite of humans and other mammals in the Americas. Ann Trop Med Parasitol. 2004:98:283-92.

2. Nava S, Beati L, Labruna MB, Cáceres AG, Mangold AJ, Guglielmone AA. Reassessment of the taxonomic status of Amblyomma cajennense (Fabricius, 1787) with the description of three new species, Amblyomma tonelliae $\mathrm{n}$. sp., Amblyomma interandinum n. sp. and Amblyomma patinoi n. sp., and reinstatement of Amblyomma mixtum Koch, 1844, and Amblyomma sculptum Berlese, 1888 (Ixodida: Ixodidae). Ticks Tick Borne Dis. 2014;5: 252-76

3. Labruna MB, Soares JF, Martins TF, Soares HS, Cabrera RR. Cross-mating experiments with geographically different populations of Amblyomma cajennense (Acari: Ixodidae). Exp Appl Acarol. 2011:54:41-9.

4. Mastropaolo M, Nava S, Guglielmone AA, Mangold AJ. Biological differences between two allopatric populations of Amblyomma cajennense (Acari: Ixodidae) in Argentina. Exp Appl Acarol. 2011;53:371-5.

5. Beati L, Nava S, Burkman EJ, Barros-Battesti D, Labruna MB, Guglielmone AA, et al. Amblyomma cajennense (Fabricius, 1787) (Acari: Ixodidae), the Cayenne tick: phylogeography and evidence for allopatric speciation. BMC Evol Biol. 2013;13:267.

6. Cooley RA, Kohls GM. The genus Amblyomma (Ixodidae) in the United States. J Parasitol. 1944;30:77-111.

7. Martins TF, Onofrio VC, Barros-Battesti DM, Labruna MB. Nymphs of the genus Amblyomma (Acari: Ixodidae) of Brazil: descriptions, redescriptions, and identification key. Ticks Tick Borne Dis. 2010;1:75-99.

8. Martins TF, Labruna MB, Mangold AJ, Cafrune MM, Guglielmone AA, Nava S. Taxonomic key to nymphs of the genus Amblyomma (Acari: Ixodidae) in Argentina, with description and redescription of the nymphal stage of four Amblyomma species. Ticks Tick Borne Dis. 2014;5:753-70.

9. Labruna MB, Terassini FA, Camargo LM. Notes on population dynamics of Amblyomma ticks (Acari: Ixodidae) in Brazil. J Parasitol. 2009;95:1016-8.

10. Labruna MB, Kasai N, Ferreira F, Faccini JL, Gennari SM. Seasonal dynamics of ticks (Acari: Ixodidae) on horses in the state of São Paulo Brazil. Vet Parasitol. 2002;19:65-77.

11. Aragão H. Ixodidas brasileiros e de alguns paizes limitrophes. Mem Inst Oswaldo Cruz. 1936:31:759-843.

12. Labruna MB, Kerber CE, Ferreira F, Faccini JL, De Waal DT, Gennari SM. Risk factors to tick infestations and their occurrence on horses in the state of São Paulo Brazil. Vet Parasitol. 2001:97:1-14.

13. Terassini FA, Barbieri FS, Albuquerque S, Szabó MP, Camargo LM, Labruna MB. Comparison of two methods for collecting free-living ticks in the Amazonian forest. Ticks Tick Borne Dis. 2010;1:194-6.

14. Sangioni LA, Horta MC, Vianna MCB, Gennari SM, Soares RM, Galvão MAM, Schumaker TTS, Ferreira F, Vidotto O, Labruna MB. Rickettsial infection in animals and Brazilian spotted fever endemicity. Emerg Infect Dis. 2005;11: 265-70. 
15. Labruna MB, Marrelli MT, Heinemann JM, Fava AB, Cortez A, Soares RM, et al. Taxonomic status of Ixodes didelphidis (Acari: Ixodidae). J Med Entomol. 2002;39:135-42.

16. Thompson JD, Gibson TJ, Plewniak F, Jeanmougin F, Higgins DG. The CLUSTAL_X Windows interface: flexible strategies for multiple sequence alignment aided by quality analysis tools. Nucleic Acids Res. 1997;25:4876-82.

17. Nicholas KB, Nicholas Jr HB. GeneDoc: analysis and visualization of genetic variation. EMBNEW News. 1997:4:14

18. Swofford DL. PAUP: Phylogenetic analysis using parsimony. Beta Version 4 Ob10. Sunderland, Massachusetts: Sinauer and Associates; 2002.

19. Huelsenbeck JP, Ronquist F. MrBayes: Bayesian inference of phylogenetic trees. Bioinformatics. 2001;17:754-5.

20. Corwin DC, Clifford CM, Keirans JE. An improved method for cleaning and preparing ticks for examination with the scanning electron microscope. J Med Entomol. 1979;16:352-3.

21. Labruna MB, Camargo LM, Terrassini FA, Ferreira F, Schumaker TTS, Camargo EP. Ticks (Acari: Ixodidae) from the state of Rondônia, western Amazon Brazil. Syst Appl Acarol. 2005;10:17-32.

22. Estrada-Peña A, Tarragona EL, Vesco U, Meneghi D, Mastropaolo M, Mangold AJ, et al. Divergent environmental preferences and areas of sympatry of tick species in the Amblyomma cajennense complex (Ixodidae). Int J Parasitol. 2014:44:1081-9.

23. Szabó MP, Labruna MB, Garcia MV, Pinter A, Castagnolli KC, Pacheco RC, et al. Ecological aspects of the free-living ticks (Acari: Ixodidae) on animal trails within Atlantic rainforest in south-eastern Brazil. Ann Trop Med Parasitol. 2009:103:57-72

24. Morellato LPC, Haddad CFB. Introduction: The Brazilian Atlantic Forest. Biotropica. 2000;32:786-92.

25. Souza C Jr, Siqueira J, Ribeiro J, Sales M (2013) Desmatamento e Degradação Florestal do Bioma Amazônia (2000-2010). Belém, Imazon, p 2

26. Krawczak FS, Nieri-Bastos FA, Nunes FP, Soares JF, Moraes-Filho J, Labruna MB. Rickettsial infection in Amblyomma cajennense ticks and capybaras (Hydrochoerus hydrochaeris) in a Brazilian spotted fever-endemic area. Parasit Vectors. 2014;7:7

27. Soares HS, Barbieri AR, Martins TF, Minervino AH, de Lima JT, Marcili A, et al. Ticks and rickettsial infection in the wildlife of two regions of the Brazilian Amazon. Exp Appl Acarol. 2015;65:125-40

28. Guglielmone AA, Beati L, Barros-Battesti DM, Labruna MB, Nava S, Venzal JM, et al. Ticks (Ixodidae) on humans in South America. Exp Appl Acarol. 2006; 40:83-100.

\section{Submit your next manuscript to BioMed Central and we will help you at every step:}

- We accept pre-submission inquiries

- Our selector tool helps you to find the most relevant journal

- We provide round the clock customer support

- Convenient online submission

- Thorough peer review

- Inclusion in PubMed and all major indexing services

- Maximum visibility for your research

Submit your manuscript at www.biomedcentral.com/submit 